\title{
Quantitative Analysis of Shock Wave Dynamics in a Fluid of Light
}

\author{
T. Bienaimé, ${ }^{1}$ M. Isoard ${ }^{2}, 3$ Q. Fontaine, ${ }^{1}$ A. Bramati, ${ }^{1}$ A. M. Kamchatnov, ${ }^{4,5}$ Q. Glorieux, ${ }^{1}$ and N. Pavloff ${ }^{2}$ \\ ${ }^{1}$ Laboratoire Kastler Brossel, Sorbonne Université, CNRS, \\ ENS-PSL Research University, Collège de France, Paris 75005, France \\ ${ }^{2}$ Université Paris-Saclay, CNRS, LPTMS, 91405, Orsay, France \\ ${ }^{3}$ Physikalisches Institut, Albert-Ludwigs-Universität Freiburg, \\ Hermann-Herder-Straße 3, D-79104 Freiburg, Germany \\ ${ }^{4}$ Moscow Institute of Physics and Technology, Institutsky lane 9, Dolgoprudny, Moscow region, 141700, Russia \\ ${ }^{5}$ Institute of Spectroscopy, Russian Academy of Sciences, Troitsk, Moscow, 108840, Russia
}

\begin{abstract}
We report on the formation of a dispersive shock wave in a nonlinear optical medium. We monitor the evolution of the shock by tuning the incoming beam power. The experimental observations for the position and intensity of the solitonic edge of the shock, as well as the location of the nonlinear oscillations are well described by recent developments of Whitham modulation theory. Our work constitutes a detailed and accurate benchmark for this approach. It opens exciting possibilities to engineer specific configurations of optical shock wave for studying wave-mean flow interaction.
\end{abstract}

In many different fields such as acoustics [1], plasma physics [2], hydrodynamics [3-5], nonlinear optics [6], ultracold quantum gases [7-10], the short time propagation of slowly varying nonlinear pulses can be described discarding the effects of dispersion and dissipation. The prototype of such an approach is given by the system of equations governing compressible gas dynamics [11]. This type of treatment typically predicts that, due to nonlinearity, an initially smooth pulse steepens during its time evolution, eventually reaching a point of gradient catastrophe. This is the wave-breaking phenomenon, which results in the formation of a shock wave $[12,13]$. If, after wave breaking, dispersive effects dominate over viscosity, the shock eventually acquires a stationary nonlinear oscillating structure for which the width increases with diminishing dissipation [14]. In the case of weak dissipation the time for reaching a stationary regime can be quite long. Gurevich and Pitaevskii [15] made a major contribution to the field when they first realized the interest of studying the evolution of the associated dynamical structure, now called a dispersive shock wave (DSW). Besides, they understood that a DSW can be described as a modulated nonlinear traveling wave and studied in the framework of the Whitham theory of modulations [16].

In the present work we study the propagation of an optical beam in a nonlinear defocusing medium. Wavebreaking and (spatial or temporal) dispersive shocks have already been observed in such a setting [17-30]. However, all previous theoretical descriptions of experimental optical shocks either remained only qualitative or resorted to numerical simulations for reaching accurate descriptions. Indeed, a realistic quantitative characterization of the experimental situation requires to take into account a number of nontrivial effects which sum up to a quite difficult task. For instance, saturation effects, such as occurring in semiconductor doped glasses [31] and in photorefractive media [32], can only be taken into account by using a nonintegrable nonlinear equation, even for a medium with a local nonlinearity. Besides, both "Rie- mann invariants" typically vary during the prebreaking period and this complicates the description of the nondispersive stage of the pulse spreading, even in a quasi unidimensional (1D) geometry. Moreover, for realistic initial intensity pulse profiles, the post-breaking evolution corresponds, at best, to a so-called "quasisimple" dispersive shock [33], the characterization of which requires an elaborate extension of the Gurevich-Pitaevskii scheme. Finally, the nonintegrability of the wave equation significantly complicates the post-breaking description of the nonlinear oscillations within the shock. Despite these difficulties, it has recently become possible to combine several theoretical advances [34-45] to obtain a comprehensive treatment of the nonlinear pulse spreading and the subsequent formation of a dispersive shock in a realistic setting $[46,47]$. In this Letter, we provide a nonambiguous experimental evidence for the accuracy of this theory with a precise description of the main features of the shock. This universal and quantitative benchmark is a major advance for manipulation and engineering of optical shockwaves.

We study the propagation of a laser field in a $L=$ $7.5 \mathrm{~cm}$-long cell filled with an isotopically pure ${ }^{85} \mathrm{Rb}$ vapor (99\% purity) warmed up to a controlled temperature of $120^{\circ} \mathrm{C}$ to adjust the atomic vapor density. We use a Ti:sapphire laser detuned by $-3.9 \mathrm{GHz}$ with respect to the $F=3 \rightarrow F^{\prime}$ transition of the D2-line of ${ }^{85} \mathrm{Rb}$ at $\lambda_{0}=2 \pi / k_{0}=780 \mathrm{~nm}$. For such a large detuning, the natural Lorentzian shape of the line dominates and the Doppler broadening $k_{0} v \simeq 240 \mathrm{MHz}$ can be safely neglected. In these experimental conditions, the system is self defocusing (repulsive photon-photon interaction) and the transmission through the cell is $60 \%$. We find that this medium is well described by local photon-photon interactions, but contrary to previous works [48-52] we find it important to take into account the saturation of the nonlinearity to quantitatively describe the dynamics of the shock waves.

The input intensity profile is a cross-beam configura- 


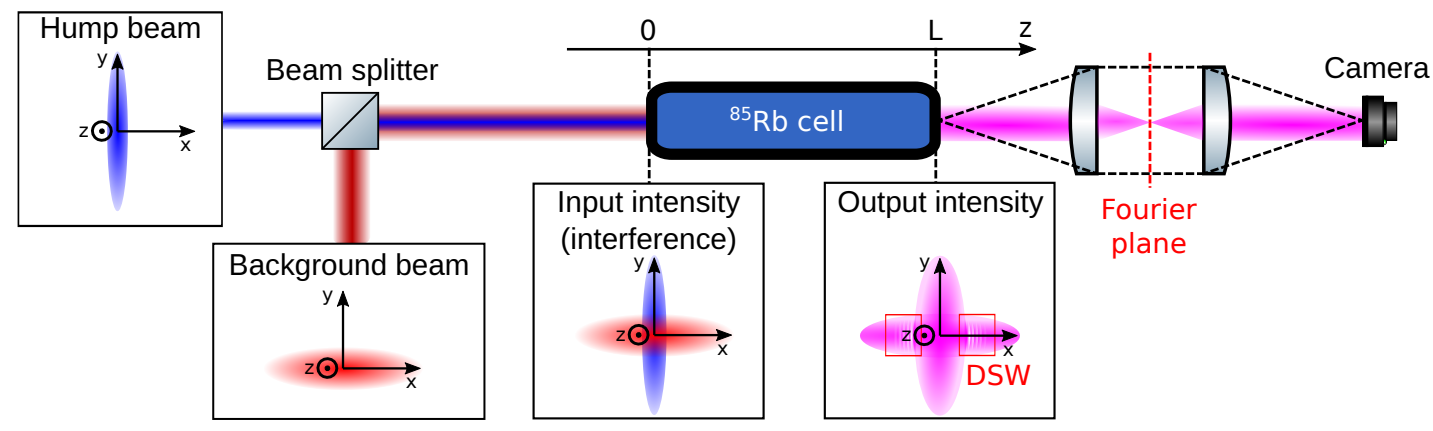

FIG. 1. Sketch of the experimental setup. To create the initial state we overlap the background and the hump beams on a beam splitter with their relative phase precisely adjusted such that they interfere constructively. This state then propagates inside the nonlinear medium consisting of a hot ${ }^{85} \mathrm{Rb}$ vapor cell of length $L$. The insets represent cuts of the relevant intensity profiles in the plane perpendicular to the direction $z$ of propagation. The output intensity is recorded on a camera by direct imaging through two lenses in $4 f$ configuration.

tion of two vertically polarized laser beams, both propagating along the axis of the cell (denoted as $\mathrm{O} z$ ), with their respective phase precisely adjusted such that the two beams interfere constructively, see Fig. 1. One of the beams (which is denoted the hump) is extended along the $y$ direction and significantly more intense than the other one (the background) which is extended along the $x$ direction. At the entrance of the cell $(z=0)$ both beams have an elliptic Gaussian profile. The background beam has a power $P_{0}$ and waists $w_{x, 0}>w_{y, 0}$, whereas the hump has power $P_{1}$ and waists $w_{x, 1}<w_{y, 1}$; see Supplemental Material [53]. During the initial nondiffractive stage of evolution, nonlinearity acts as an effective pressure which favors spreading of the hump in the $x$ direction along which is initially tighter collimated. Conversely, the low intensity background experiences almost no spreading and behaves as a pedestal which triggers wave breaking of the hump during its spreading. Each beam has a maximum entrance intensity $\mathcal{I}_{\alpha}=2 P_{\alpha} /\left(\pi w_{x, \alpha} w_{y, \alpha}\right)(\alpha=0$ or 1$)$, and we explore the DSW dynamics for a fixed ratio $\mathcal{I}_{1} / \mathcal{I}_{0}$. We work in the deep nonlinear regime, with $\mathcal{I}_{1}=20 \mathcal{I}_{0}$. This large value corresponds to a wave breaking distance typically shorter than the cell length, and makes it possible, by changing the total power $P_{\text {tot }}$ of the beams, to observe several stages of evolution of the DSW. The total power can be increased up to $700 \mathrm{~mW}$ and is limited by the laser maximum output power.

We image the total field intensity $I_{\text {out }}(x, y)$ at the output of the cell on a camera. In order to minimize the effect of absorption and increase the visibility of the DSW, we determine the normalized output intensity

$$
\widetilde{I}_{\text {out }}(x, y) \equiv \frac{I_{\text {out }}(x, y)-I_{\text {out }}^{0}(x, y)}{\mathcal{I}_{\text {out }}^{0}},
$$

where $I_{\text {out }}^{0}(x, y)$ is the intensity profile at the cell output when only the background beam propagates through the medium (the hump beam is blocked). $\mathcal{I}_{\text {out }}^{0}$ is the maximal value of $I_{\text {out }}^{0}(x, y) . \widetilde{I}_{\text {out }}(x, y)$ is represented in Fig. 2(a). Our theoretical description relies on only two parameters which characterize the photon-photon interaction, namely, the Kerr coefficient, $n_{2}$, and the saturation intensity $I_{\text {sat }}$ [cf. Eq. (2)]. Their values $n_{2}=1.5 \times 10^{-4}$ $\mathrm{mm}^{2} / \mathrm{W}$, and $I_{\text {sat }}=0.6 \mathrm{~W} / \mathrm{mm}^{2}$ have been determined by comparing the experimental results with large-scale $2 \mathrm{D}$ numerical simulations [53]. The excellent agreement reached in Fig. 2 indicates that two effects - saturable nonlinearity and linear absorption - are the relevant physical ingredients for a theoretical description of our experiment.

In the regime $w_{1, x} \ll w_{0, x}$ and $\mathcal{I}_{1} \gg \mathcal{I}_{0}$ we consider, the normalized output density $\widetilde{I}_{\text {out }}$ becomes independent on the precise shape of the background beam. As a result, $\widetilde{I}_{\text {out }}(x, 0)$ can be described by using a simplified 1D theoretical description, where a hump propagates over a background of uniform intensity $\mathcal{I}_{0}$. Within the cell, the complex field amplitude at $y=0$, denoted as $\mathcal{A}(x, 0, z) \equiv a(x, z)$, then obeys a $1 \mathrm{D}$ nonlinear Schrödinger equation where the position $z$ along the axis of the beam plays the role of an effective "time" [54]. The equation, once included the nonlinearity saturation and the linear absorption [32], reads

$$
i \partial_{z} a=-\frac{1}{2 n_{0} k_{0}} \partial_{x}^{2} a+\frac{k_{0} n_{2}|a|^{2}}{1+|a|^{2} / I_{\mathrm{sat}}} a-\frac{i}{\Lambda_{\mathrm{abs}}} a,
$$

where $n_{0} \simeq 1$ is the linear index of refraction and $\Lambda_{\text {abs }}=$ $30 \mathrm{~cm}$, which corresponds to a $60 \%$ transmission for a cell of length $L=7.5 \mathrm{~cm}$. The value of the effective amplitude at the entrance of the cell is taken as

$$
a(x, 0)=\sqrt{\mathcal{I}_{0}}+\sqrt{\mathcal{I}_{1}} \exp \left(-\frac{x^{2}}{w_{x, 1}^{2}}\right),
$$

In order to evaluate the accuracy of the mapping to the 1D model of Eq. (2), we compare in the upper panel of Fig. 2(b) the corresponding value of $|a(x, L)|^{2} \exp \left(2 L / \Lambda_{\text {abs }}\right) / \mathcal{I}_{0}-1$ with the experimental 


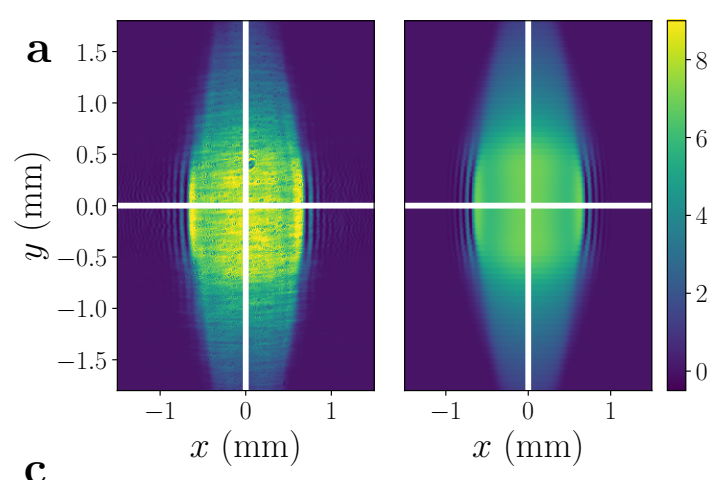

C
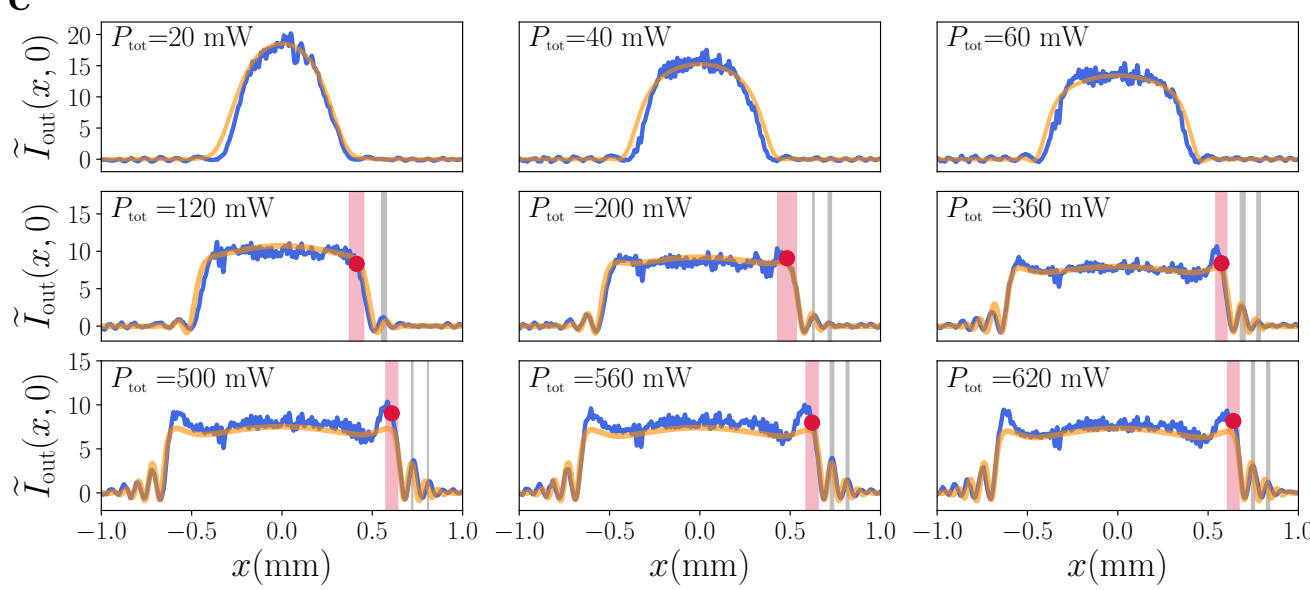
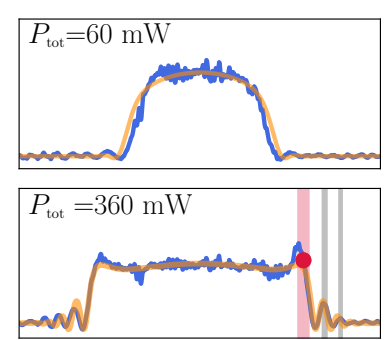
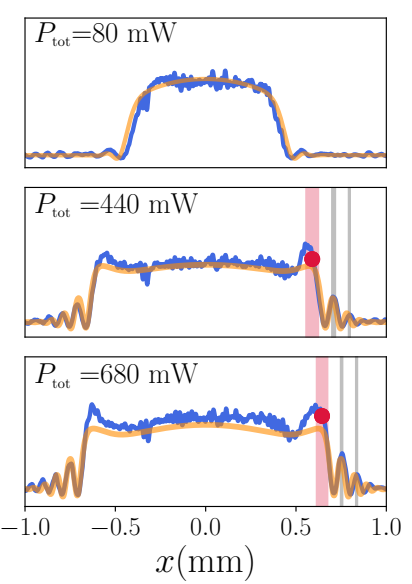

FIG. 2. (a) Left: experimental profile $\widetilde{I}_{\text {out }}$ taken for $P_{\text {tot }}=680 \mathrm{~mW}$. Right: Two-dimensional numerical simulations at the same total entrance power. (b) $x$ and $y$ profiles along the cuts represented by the two white lines on the two-dimensional profiles (a). The solid blue line represents the experimental data, the dashed green line the two-dimensional numerical simulation. On the $x$ profile, the orange line is a one-dimensional numerical simulation, from Eqs. (2) and (3). (c) $\widetilde{I}_{\text {out }}(x, 0)$ for various total beam powers. The color code is the same as in (b). The vertical pink and gray bars on the right part of each intensity profile indicate the positions of the solitonic edge of the DSW and of the first maxima of oscillations within the DSW. The thickness of each bar represents the experimental uncertainty.

$\widetilde{I}_{\text {out }}(x, 0)$ and with the result of $2 \mathrm{D}$ simulations. The excellent agreement is confirmed in Fig. 2(c) for the whole range of beam powers $P_{\text {tot }}$.

The mapping to a $1 \mathrm{D}$ problem enables us to compare our measurements with recent analytical predictions. In particular, if one neglects the linear absorption within the cell, for the initial intensity profile (3), wave breaking occurs at a propagation distance [45]

$$
z_{\mathrm{WB}}=4 \sqrt{\frac{n_{0} I^{*}}{n_{2}}} \frac{\left(1+I^{*} / I_{\mathrm{sat}}\right)^{2}}{3+I^{*} / I_{\mathrm{sat}}} \cdot \frac{1}{\max \left|\frac{d I(x, 0)}{d x}\right|},
$$

where $I(x, 0)=|a(x, 0)|^{2}$ is the entrance intensity and $I^{*}$ is the value $I\left(x^{*}, 0\right)$ at point $x^{*}$ where $|d I(x, 0) / d x|$ reaches its maximum. For low entrance power, no DSW is observed because $z_{\mathrm{WB}}$ is larger than the cell length. Wave breaking first occurs within the cell for a total power $P_{\mathrm{wв}}$ such that $z_{\mathrm{WB}}=L$. For our experimental parameters we obtain $P_{\mathrm{wB}}=48 \mathrm{~mW}$. Numerical tests show that taking absorption into account does not modify notably this value.
For a total power larger than $P_{\mathrm{wB}}$, the DSW is formed and develops within the cell. The physical phenomenon at the origin of the DSW is the following: large intensity perturbations propagate faster than small ones, so there exist values of $x$ reached at the same "time" by different intensities. When this occurs first, the density gradient is infinite. This corresponds to the onset of a cusp catastrophe [55, 56], the nonlinear diffractive dressing of which is a dispersive shock wave. This takes the form of a modulated oscillating pattern consisting asymptotically (i.e. at large $z$, or equivalently large $P_{\text {tot }}$ ) in a train of solitons which, away from the center of the beam, gradually evolves into a linear perturbation. The position of its "solitonic edge" on the $y=0$ axis at the cell output $(z=L)$ is denoted as $x_{s}$. It is located in Figs. 2(c) by a vertical red bar whose thickness represents the uncertainty on the estimation of $x_{s}$ from the experimental $\widetilde{I}_{\text {out }}(x, 0)$. This uncertainty limits the experimental determination of $x_{s}$ to powers larger than $120 \mathrm{~mW}$. The following maxima of oscillations, represented by vertical gray lines, are more precisely determined experimentally. 

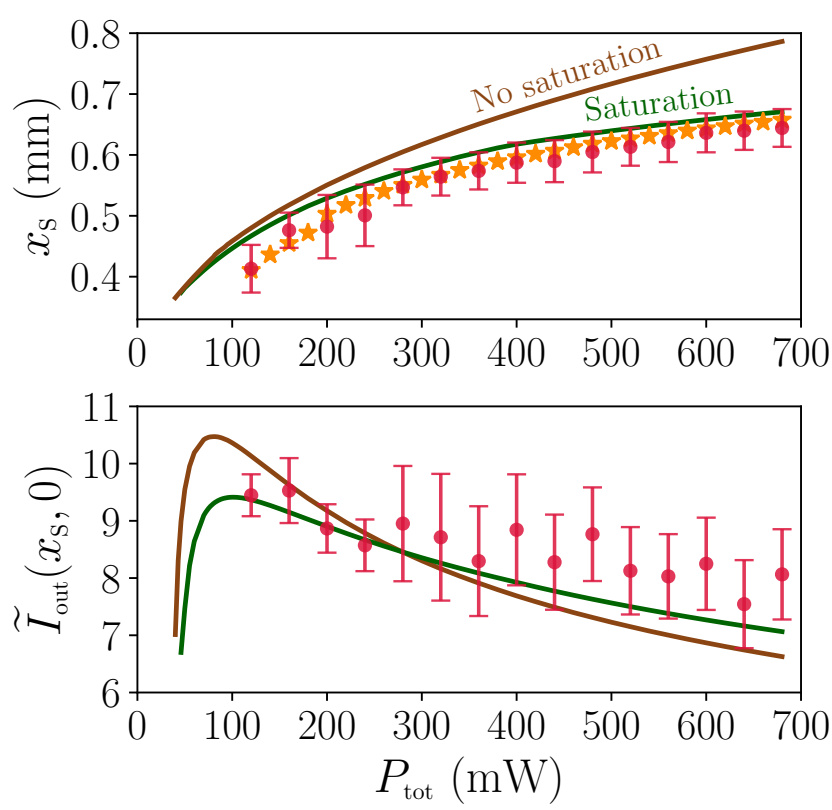

FIG. 3. Characterization of the solitonic edge of the DSW as a function of the beam's power. The upper panel represents the position $x_{s}$ of the shock, and the lower one the corresponding intensity $\widetilde{I}_{\text {out }}\left(x_{s}, 0\right)$. In each panel, the red points with error bars are experimental results, from Fig. 2(c) and, in the upper one, the orange stars are the results of $1 \mathrm{D} \mathrm{nu-}$ merical simulations of Eq. (2). The green solid line is the theoretical result, from Ref. [47]. The brown solid line is the theoretical result in the absence of saturation.

The technique devised in Ref. [47] makes it possible to theoretically determine $x_{s}$ and the corresponding intensity $\widetilde{I}_{\text {out }}\left(x_{s}, 0\right)$. As illustrated in Fig. 3 the results of this analytical approach (green solid lines) compare well with the experimental data, although it does not take absorption into account. Importantly, omission of the nonlinearity saturation leads to incorrect results (brown solid line).

One may study the DSW in an even more detailed way by locating the position of the maxima of the nonlinear oscillations. While the theoretical results for $x_{s}$ essentially rely on an approach due to El [34, 40, 43] which is valid for any type of nonlinearity, the precise intensity profile within the DSW can be computed only for exactly integrable systems, i.e., by neglecting saturation effects. The position-dependent oscillation period $\mathcal{L}(x, z)$ was computed in this framework in Ref. [46] for a parabolic initial intensity distribution. Fitting the center of the intensity profile (3) by an inverted parabola, the positions $x_{1}, x_{2}$, and $x_{3}$ of the first maxima of oscillation of the DSW at the output of the cell are determined by

$$
x_{1}-x_{s}=\mathcal{L}\left(\frac{x_{s}+x_{1}}{2}, L\right)
$$

and by similar formulas obtained by replacing $x_{1}$ by $x_{2}$ (then $x_{3}$ ) and $x_{s}$ by $x_{1}$ (then $x_{2}$ ). The results are

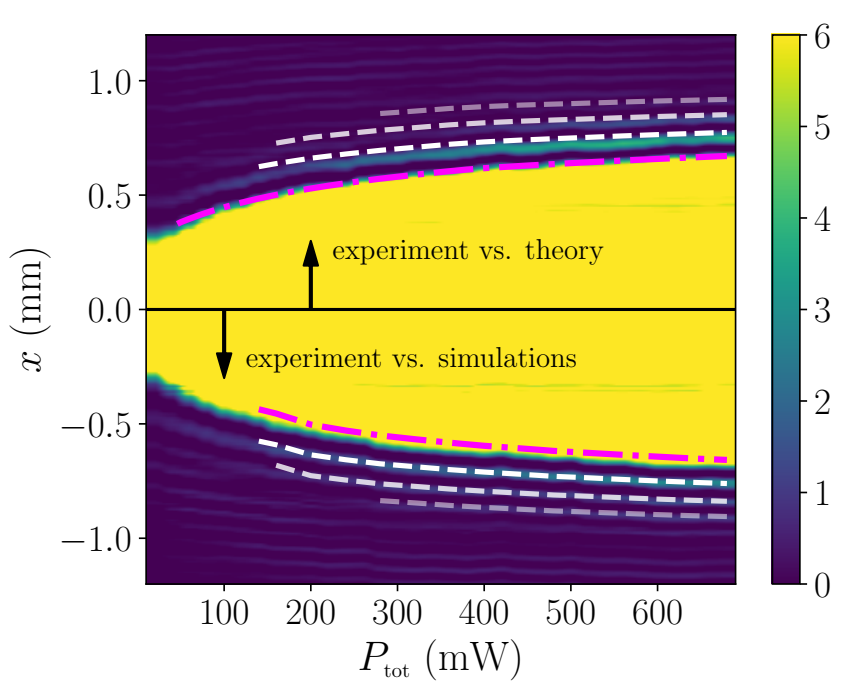

FIG. 4. Color plot of the experimental intensity profiles $\widetilde{I}_{\text {out }}(x, 0)$ as a function of $P_{\text {tot. }}$. The purple dot-dashed line represents the edge $x_{s}$ of the DSW extracted from Whitham theory (upper part of the figure: $x>0$ ) and from 1D numerical simulations (lower part, $x<0$ ). In each half of the figure $(x \lessgtr 0)$ the white dashed lines are the corresponding analytic predictions (5) for the maxima of oscillation.

compared with the experimental ones in the upper half $(x>0)$ of Fig. 4. The small offset in the position of the theoretical maxima with respect to the experimental ones observed in the figure is due to an initial small overshoot in the theoretical position of $x_{s}$ (cf. the green solid line in Fig. 3) which is itself due to the absence of absorption in the model. Indeed, the 1D numerical simulations - which do take absorption into account - are in slightly better agreement with the experimental results for $x_{s}$ (cf. the orange stars in Fig. 3). Using the numerical $x_{s}$ in Eq. (5) instead of the analytical one yields, for the maxima of oscillations, an excellent agreement with experiment, cf. the lower half of Fig. 4. Such a good agreement despite the fact that Eq. (5) does not take saturation into account is not surprising: the rapid decrease of intensity away from the solitonic edge (cf. Fig. 2) significantly reduces the importance of saturation within the DSW.

It thus appears possible to give a detailed description of precise experimental recordings of the intensity pattern of an optical shock wave, not only thanks to numerical simulations, but on the basis of Whitham's modulation theory. This is an important validation of recent advances in this approach, which is no longer restricted to integrable systems or idealized initial configurations. We are reaching a point where these progresses make it possible not only to study DSWs per se, but also as tools for prospecting new physical phenomena, such as the type of wave-mean flow interaction recently identified in Ref. [57]: our platform is ideally designed to investigate scattering of elementary excitations by a DSW, a 
study which is also relevant to the domain of analogue gravity. Indeed, as discussed in the Supplemental Material [53], a dispersive shock can be considered as an exotic model of acoustic white hole, and the good experimental control and theoretical understanding of this structure demonstrated in the present work opens the prospect of a detailed investigation of the corresponding induced background fluctuations.

[1] S. N. Gurbatov, O. V. Rudenko, and A. I. Aaichev, Waves and Structures in Nonlinear Nondispersive Media, General Theory and Applications to Nonlinear Acoustics (Springer-Verlag, Berlin and Heidelberg, 2011).

[2] A. Jeffrey and T. Taniuti, Non-Linear Wave Propagation with Applications to Physics and Magnetohydrodynamics (Academic Press, London, 1964).

[3] A. J. C. Barré de Saint-Venant, Théorie du mouvement non permanent des eaux, avec application aux crues des rivières et à l'introduction de marées dans leurs lits, C. R. Acad. Sci. Paris 73, 147 and 237 (1871).

[4] H. Lacombe and M. Bellevaux, Courants de densité dans le détroit de Gibraltar, La Houille Blanche 1, 38 (1965).

[5] L. V. Ovsyannikov, Two-layer "shallow water" model, J. Appl. Mech. Tech. Phys. 20, 127 (1979).

[6] S. A. Akhmanov, A. P. Sukhorukov, and R. V. Khokhlov, Self-focusing and self-trapping of intense light beams in a nonlinear medium, Sov. Phys. JETP 25, 1025 (1966).

[7] Y. Kagan, E. L. Surkov, and G. V. Shlyapnikov, Evolution of a Bose-condensed gas under variations of the confining potential, Phys. Rev. A 54, R1753 (1996).

[8] Y. Castin and R. Dum, Bose-Einstein condensates in time dependent traps, Phys. Rev. Lett. 77, 5315 (1996).

[9] J. A. Joseph, J. E. Thomas, M. Kulkarni, and A. G. Abanov, Observation of shock waves in a strongly interacting Fermi gas, Phys. Rev. Lett. 106, 150401 (2011).

[10] S. Datta and U. R. Fischer, Fluid-dynamical analogue of nonlinear gravitational wave memory (2020), arXiv:2011.05837 [gr-qc].

[11] B. Riemann, On the propagation of planar air waves of finite amplitude, in Collected papers (Kendrick Press, 2004).

[12] R. Courant and K. O. Friedrichs, Supersonic flow and shock waves, a manual on the mathematical theory of non-linear wave motion (Scholar's Choice, 2015).

[13] Y. B. Zel'dovich and Y. P. Raizer, Physics of shock waves and high-temperature hydrodynamic phenomena (Dover, 2002).

[14] R. Z. Sagdeev, Cooperative phenomena and shock waves in collisionless plasmas, in Reviews of Plasma Physics, Vol. 4, edited by M. A. Leontovich (Consultants Bureau, New York, 1966) p. 23.

[15] A. V. Gurevich and L. P. Pitaevskii, Nonstationary structure of a collisionless shock wave, Sov. Phys. JETP 38, 291 (1973).

[16] G. B. Whitham, Non-linear dispersive waves, Proc. R. Soc. Lond. A 283, 238 (1965).

[17] J. E. Rothenberg and D. Grischkowsky, Observation of the formation of an optical intensity shock and wave breaking in the nonlinear propagation of pulses in op- tical fibers, Phys. Rev. Lett. 62, 531 (1989).

[18] G. Couton, H. Maillotte, and M. Chauvet, Self-formation of multiple spatial photovoltaic solitons, J. Opt. B 6 , S223 (2004).

[19] W. Wan, S. Jia, and J. W. Fleischer, Dispersive superfluid-like shock waves in nonlinear optics, Nat. Phys. 3, 46 (2007).

[20] S. Jia, W. Wan, and J. W. Fleischer, Dispersive shock waves in nonlinear arrays, Phys. Rev. Lett. 99, 223901 (2007).

[21] N. Ghofraniha, C. Conti, G. Ruocco, and S. Trillo, Shocks in nonlocal media, Phys. Rev. Lett. 99, 043903 (2007).

[22] C. Barsi, W. Wan, C. Sun, and J. W. Fleischer, Dispersive shock waves with nonlocal nonlinearity, Opt. Lett. 32, 2930 (2007).

[23] N. Ghofraniha, S. Gentilini, V. Folli, E. DelRe, and C. Conti, Shock waves in disordered media, Phys. Rev. Lett. 109, 243902 (2012).

[24] M. Elazar, V. Fleurov, and S. Bar-Ad, All-optical event horizon in an optical analog of a laval nozzle, Phys. Rev. A 86, 063821 (2012).

[25] N. Ghofraniha, L. Santamaria Amato, V. Folli, S. Trillo, E. DelRe, and C. Conti, Measurement of scaling laws for shock waves in thermal nonlocal media, Opt. Lett. 37, 2325 (2012).

[26] J. Fatome, C. Finot, G. Millot, A. Armaroli, and S. Trillo, Observation of optical undular bores in multiple fourwave mixing, Phys. Rev. X 4, 021022 (2014).

[27] G. Xu, A. Mussot, A. Kudlinski, S. Trillo, F. Copie, and M. Conforti, Shock wave generation triggered by a weak background in optical fibers, Opt. Lett. 41, 2656 (2016).

[28] B. Wetzel, D. Bongiovanni, M. Kues, Y. Hu, Z. Chen, S. Trillo, J. M. Dudley, S. Wabnitz, and R. Morandotti, Experimental generation of Riemann waves in optics: A route to shock wave control, Phys. Rev. Lett. 117, 073902 (2016).

[29] G. Xu, M. Conforti, A. Kudlinski, A. Mussot, and S. Trillo, Dispersive dam-break flow of a photon fluid, Phys. Rev. Lett. 118, 254101 (2017).

[30] J. Nuño, C. Finot, G. Xu, G. Millot, M. Erkintalo, and J. Fatome, Vectorial dispersive shock waves in optical fibers, Commun. Phys. 2, 138 (2019).

[31] J.-L. Coutaz and M. Kull, Saturation of the nonlinear index of refraction in semiconductor-doped glass, J. Opt. Soc. Am. B 8, 95 (1991).

[32] Y. S. Kivshar and G. P. Agrawal, Optical Solitons, From Fibers to Photonic Crystals (Academic, New York, 2003).

[33] A. V. Gurevich and A. L. Krylov, Dissipationless shock waves in media with positive dispersion, Sov. Phys. JETP 65, 944 (1987).

[34] A. V. Gurevich and A. P. Meshcherkin, Expanding selfsimilar discontinuities and shock waves in dispersive hydrodynamics, Sov. Phys. JETP 60, 732 (1984).

[35] A. V. Gurevich, A. L. Krylov, and N. G. Mazur, Quasisimple waves in Korteweg-de Vries hydrodynamics, Sov. Phys. JETP 68, 966 (1989).

[36] A. L. Krylov, V. V. Khodorovskii, and G. A. El, Evolution of a nonmonotonic perturbation in Korteweg-de Vries hydrodynamics, JETP Lett. 56, 323 (1992).

[37] G. A. El and V. V. Khodorovsky, Evolution of a solitonless large-scale perturbation in Korteweg-de Vries hydrodynamics, Phys. Lett. A 182, 49 (1993).

[38] G. A. El and A. L. Krylov, General solution of the Cauchy problem for the defocusing NLS equation in the Whitham 
limit, Phys. Lett. A 203, 77 (1995).

[39] Y. Kodama, The Whitham equations for optical communications: Mathematical theory of NRZ, SIAM J. Appl. Math. 59, 2162 (1999).

[40] G. A. El, Resolution of a shock in hyperbolic systems modified by weak dispersion, Chaos 15, 037103 (2005).

[41] G. A. El, A. Gammal, E. G. Khamis, R. A. Kraenkel, and A. M. Kamchatnov, Theory of optical dispersive shock waves in photorefractive media, Phys. Rev. A 76, 053813 (2007).

[42] M. G. Forest, C.-J. Rosenberg, and O. C. Wright, On the exact solution for smooth pulses of the defocusing nonlinear Schrödinger modulation equations prior to breaking, Nonlinearity 22, 2287 (2009).

[43] A. M. Kamchatnov, Dispersive shock wave theory for nonintegrable equations, Phys. Rev. E 99, 012203 (2019).

[44] M. Isoard, A. M. Kamchatnov, and N. Pavloff, Long-time evolution of pulses in the Korteweg-de Vries equation in the absence of solitons reexamined: Whitham method, Phys. Rev. E 99, 012210 (2019).

[45] M. Isoard, A. M. Kamchatnov, and N. Pavloff, Dispersionless evolution of inviscid nonlinear pulses, Europhys. Lett. 129, 64003 (2020).

[46] M. Isoard, A. M. Kamchatnov, and N. Pavloff, Wave breaking and formation of dispersive shock waves in a defocusing nonlinear optical material, Phys. Rev. A 99, 053819 (2019).

[47] S. K. Ivanov, J.-E. Suchorski, A. M. Kamchatnov, M. Isoard, and N. Pavloff, Formation of dispersive shock waves in a saturable nonlinear medium, Phys. Rev. E 102, 032215 (2020).

[48] N. Santić, A. Fusaro, S. Salem, J. Garnier, A. Picozzi, and R. Kaiser, Nonequilibrium precondensation of classical waves in two dimensions propagating through atomic vapors, Phys. Rev. Lett. 120, 055301 (2018).

[49] C. Michel, O. Boughdad, M. Albert, P.-E. Larré, and M. Bellec, Superfluid motion and drag-force cancellation in a fluid of light, Nat. Commun. 9, 2108 (2018).

[50] Q. Fontaine, T. Bienaimé, S. Pigeon, E. Giacobino, A. Bramati, and Q. Glorieux, Observation of the Bogoli- ubov dispersion in a fluid of light, Phys. Rev. Lett. 121, 183604 (2018).

[51] Q. Fontaine, P.-E. Larré, G. Lerario, T. Bienaimé, S. Pigeon, D. Faccio, I. Carusotto, E. Giacobino, A. Bramati, and Q. Glorieux, Interferences between Bogoliubov excitations in superfluids of light, Phys. Rev. Research 2, 043297 (2020).

[52] O. Boughdad, A. Eloy, F. Mortessagne, M. Bellec, and C. Michel, Anisotropic nonlinear refractive index measurement of a photorefractive crystal via spatial selfphase modulation, Opt. Express 27, 30360 (2019).

[53] See Supplemental Material which presents our 2D numerical simulations, discusses the analogy of a dispersive shock with a white hole, and includes Refs. [56-62].

[54] L. D. Landau and E. M. Lifshitz, Electrodynamics of Continuous Media, Volume 8 (Course of Theoretical Physics) (Pergamon Press, 1984).

[55] M. Golubitsky, An introduction to catastrophe theory and its applications, SIAM Rev. 20, 352 (1978).

[56] A. M. Kamchatnov, Nonlinear Periodic Waves and Their Modulations - An Introductory Course (World Scientific, Singapore, 2000).

[57] T. Congy, G. A. El, and M. A. Hoefer, Interaction of linear modulated waves and unsteady dispersive hydrodynamic states with application to shallow water waves, J. Fluid Mech. 875, 1145 (2019).

[58] G. El, V. Geogjaev, A. Gurevich, and A. Krylov, Decay of an initial discontinuity in the defocusing NLS hydrodynamics, Physica D 87, 186 (1995).

[59] A. V. Gurevich and L. P. Pitaevskii, Averaged description of waves in the Korteweg-de Vries-Burgers equation, Sov. Phys. JETP 66, 409 (1987).

[60] V. V. Avilov, I. M. Krichever, and S. P. Novikov, Evolution of Whitham zone in the theory of Korteweg-de Vries, Sov. Phys. Dokl. 32, 564 (1987).

[61] A. Kamchatnov, On Whitham theory for perturbed integrable equations, Physica D 188, 247 (2004).

[62] P.-E. Larré, N. Pavloff, and A. M. Kamchatnov, Wave pattern induced by a localized obstacle in the flow of a one-dimensional polariton condensate, Phys. Rev. B 86, 165304 (2012). 


\section{Supplemental Material}

\section{TWO-DIMENSIONAL NUMERICAL SIMULATIONS}

The amplitudes of the incoming beams are described by the complex fields $\mathcal{A}_{\alpha}(x, y, z=0)$ where $\alpha=0$ for the background and $\alpha=1$ for the hump. Their explicit expressions read

$$
\mathcal{A}_{\alpha}(x, y, z=0)=\sqrt{\mathcal{I}_{\alpha}} \exp \left(-\frac{x^{2}}{w_{x, \alpha}^{2}}-\frac{y^{2}}{w_{y, \alpha}^{2}}\right),
$$

where the values of the different waists are $w_{x, 0}=$ $3.35 \mathrm{~mm}, w_{y, 0}=0.4 \mathrm{~mm}, w_{x, 1}=0.23 \mathrm{~mm}$ and $w_{y, 1}=1.65 \mathrm{~mm}$. The total field at the entrance of the cell is then given by $\mathcal{A}(x, y, 0)=\mathcal{A}_{0}(x, y, 0)+$ $\mathcal{A}_{1}(x, y, 0)$; it corresponds to a total incident power $P_{\text {tot }}=\int \mathrm{d} x \mathrm{~d} y|\mathcal{A}(x, y, 0)|^{2}$. From expressions (S1) one obtains

$$
\frac{P_{\text {tot }}}{P_{0}}=1+\beta \frac{w_{x, 1} w_{y, 1}}{w_{x, 0} w_{y, 0}}+4 \sqrt{\beta \frac{w_{x, 1} w_{y, 1}}{w_{x, 0} w_{y, 0}}},
$$

where $P_{0}=\int \mathrm{d} x \mathrm{~d} y\left|\mathcal{A}_{0}(x, y, 0)\right|^{2}=\frac{\pi}{2} \mathcal{I}_{0} w_{x, 0} w_{y, 0}$ is the incoming power of the background beam and $\beta=\mathcal{I}_{1} / \mathcal{I}_{0}$. In all this work we impose $\beta=20$ which corresponds to a highly nonlinear regime and favors a rapid formation of the dispersive shock wave (DSW). Within the nonlinear cell, in the paraxial approximation, the amplitude of the beam obeys a 2D generalized nonlinear Schrödinger equation:

$$
i \partial_{z} \mathcal{A}=-\frac{1}{2 n_{0} k_{0}} \vec{\nabla}_{\perp}^{2} \mathcal{A}+\frac{k_{0} n_{2}|\mathcal{A}|^{2}}{1+|\mathcal{A}|^{2} / I_{\mathrm{sat}}} \mathcal{A}-\frac{i}{\Lambda_{\mathrm{abs}}} \mathcal{A}
$$

where $\vec{\nabla}_{\perp}^{2}=\partial_{x}^{2}+\partial_{y}^{2}, n_{2}$ is the nonlinear Kerr coefficient, $I_{\text {sat }}$ the saturating density of the nonlinearity and $\Lambda_{\text {abs }}$ describes the effects of absorption. The power at the exit of our cell of length $L$ is only $60 \%$ of the incoming one, this corresponds to $\Lambda=4 L$ (i.e., $\exp \left(-2 L / \Lambda_{\mathrm{abs}}\right)=0.6$ ). Changing the value of the total incoming power $P_{\text {tot }}$ modifies the position $z_{\mathrm{wB}}$ at which wave breaking occurs and makes it possible to observe different stages of evolution of the DSW at the output $z=L$ of the nonlinear cell, see the left column of Fig. S1. We solved Eq. (S2) by two independent numerical methods (the split step method and the fourth order Runge-Kutta technique) for different values of $P_{\text {tot }}$. The best agreement with the experimental profiles is obtained for the choice of parameters $n_{2}=1.5 \times 10^{-4} \mathrm{~mm}^{2} / \mathrm{W}$ and $I_{\text {sat }}=0.6 \mathrm{~W} / \mathrm{mm}^{2}$. Fig. $\mathrm{S} 1$ compares the normalized intensity profiles obtained experimentally and numerically. It reveals very good quantitative agreement between the two.

Another test of the relevance, over all the range of input powers, of our choice of parameters is the comparison of the experimental result for the normalized output intensity $\widetilde{I}_{\text {out }}(x, y=0)$ with the ones of numerical simulations. This check is performed in Fig. S2, in which one can observe an overall agreement for the front $x_{s}$ of the shock (interface between the dark and yellow regions) and also for the location of the first maxima of oscillation whithin the dispersive shock wave. The agreement is very good for all incident powers (from $20 \mathrm{~mW}$ up to $680 \mathrm{~mW})$.

\section{A DISPERSIVE SHOCK WAVE CONSIDERED AS A WHITE HOLE}

In this section, we argue that a dispersive shock wave realizes a transsonic flow which can be considered as an implementation of an analog white hole. For simplying the presentation, we do not take into account absorption or saturation of nonlinearity: this amounts to take $I_{\text {sat }}=\Lambda_{\text {sat }}=\infty$ in a $1 \mathrm{D}$ version of Eq. (S2). Also, we do not discuss the complicated situation considered in the main text, but rather the ideal case of a perfect Gurevitch-Pitaevskii configuration issued from Riemann initial conditions, with piece-wise initial intensity and velocity, discontinuous at $x=0$, see e.g., Ref. [1]. In such a configuration the intensity and the Riemann invariants behave as sketched in Fig. S3, where the dispersive shock propagates to the right, with asymptotic intensity and velocity ${ }^{1} I_{R}$ and $u_{R}$ at the right and $I_{L}$ and $u_{L}$ at the left $\left(I_{L}>I_{R}\right.$ and $\left.u_{L}>u_{R}\right)$. Outside of the shock region, the dispersionless Riemann invariants are $\lambda_{L / R}^{ \pm}=\frac{1}{2} u_{R / L} \pm c_{R / L}$ where $c_{R / L}=\sqrt{n_{2} I_{R / L} / n_{0}}$ is the speed of sound in a uniform fluid with intensity $I_{R / L}$. Within the shock, only one of the Whitham-Riemann invariants (the colored $\lambda_{i}$ 's of Fig. S3) is not constant: $\lambda_{3}$. Its value is determined by [2]

$$
x / t=v_{3}\left(\lambda_{1}, \lambda_{2}, \lambda_{3}, \lambda_{4}\right)
$$

with $\lambda_{1}=\lambda_{R}^{-}=\lambda_{L}^{-}, \lambda_{2}=\lambda_{R}^{+}, \lambda_{4}=\lambda_{L}^{+}$and

$$
v_{3}=\frac{1}{2} \sum_{i=1}^{4} \lambda_{i}-\frac{\left(\lambda_{4}-\lambda_{3}\right)\left(\lambda_{3}-\lambda_{2}\right) \mathrm{K}(m)}{\left(\lambda_{3}-\lambda_{2}\right) \mathrm{K}(m)-\left(\lambda_{4}-\lambda_{2}\right) \mathrm{E}(m)}
$$

where

$$
m=\frac{\left(\lambda_{2}-\lambda_{1}\right)\left(\lambda_{4}-\lambda_{3}\right)}{\left(\lambda_{4}-\lambda_{2}\right)\left(\lambda_{3}-\lambda_{1}\right)}
$$

\footnotetext{
1 Strictly speaking the use of the term "velocity" is improper. Its use amounts to consider the $z$ variable as an effective time, as done in the main text.
} 

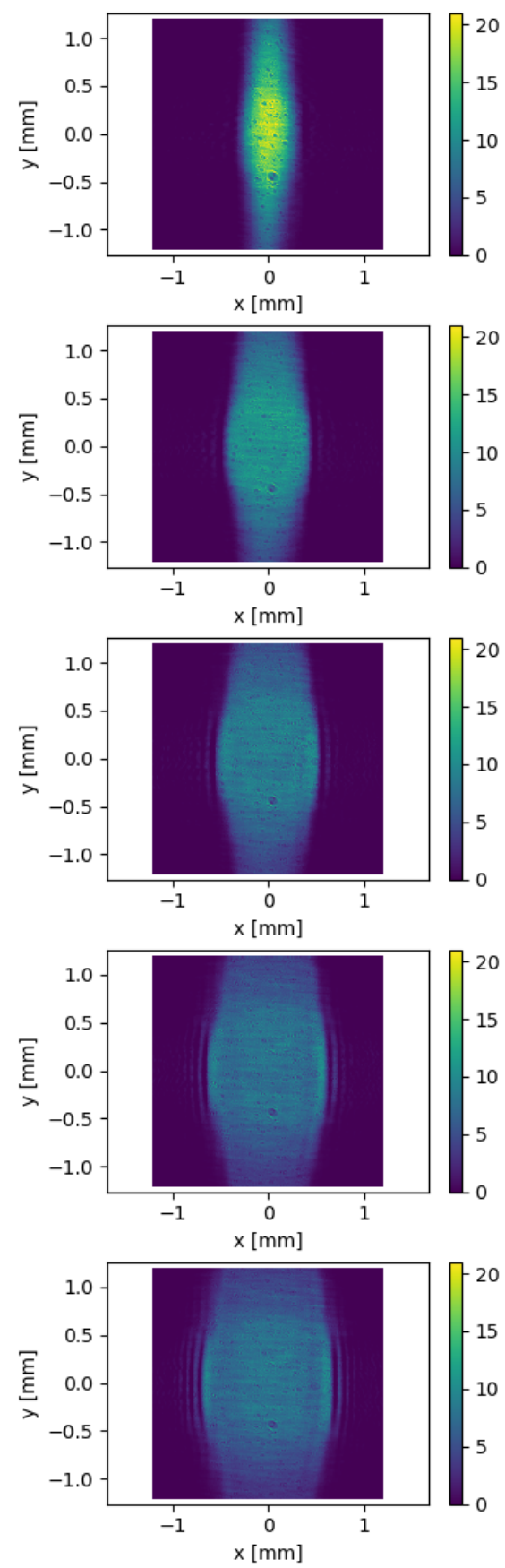
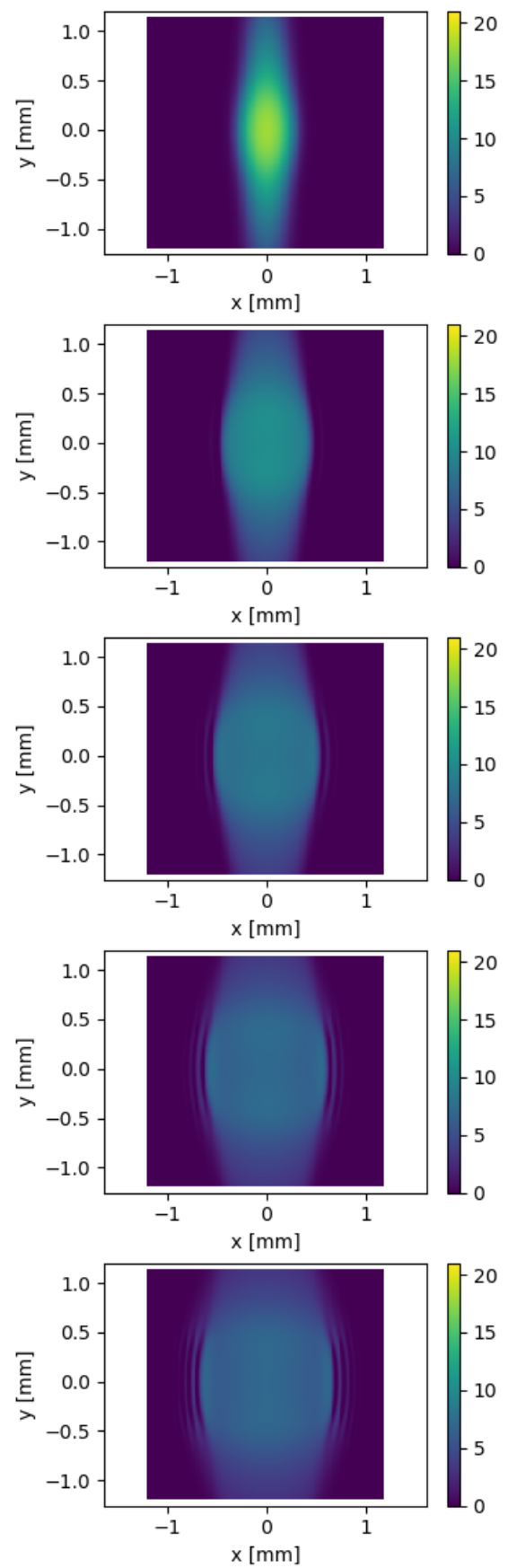
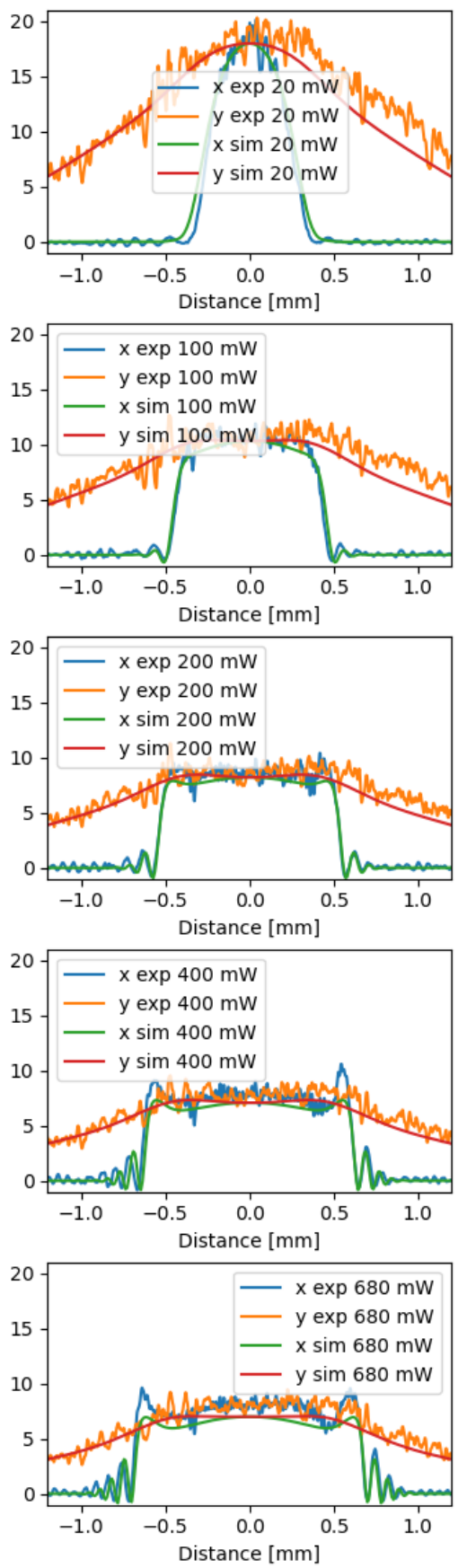

FIG. S1. Normalized intensity $\widetilde{I}_{\text {out }}(x, y)$ for the experimental data and the two-dimensional numerical simulations for $P_{\text {tot }}=$ $20,100,200,400$ and $680 \mathrm{~mW}$ from top to bottom row. The first column shows the experimental data. The second column is the corresponding numerical simulation with the parameters used in the text. The third column compares, for each total incoming power, $\widetilde{I}_{\text {out }}(x, 0)$ and $\widetilde{I}_{\text {out }}(0, y)$ for the experimental data and the simulations.

and $\mathrm{K}(m)$ and $\mathrm{E}(m)$ are the complete elliptic integrals of the first and second kind, respectively.

At the location $x=x_{s}$ of the solitonic edge, $\lambda_{2}=\lambda_{3}$ and $v_{3}$ takes the limiting value $v_{3}=\frac{1}{2}\left(\lambda_{1}+2 \lambda_{2}+\lambda_{4}\right)$ which is also the velocity $v_{s}$ of the solitonic edge. This yields

$$
v_{s}=u_{R}+c_{L} .
$$

At the opposite "harmonic edge" (of position $x_{h}$ ) one has instead $\lambda_{3}=\lambda_{4}$ and $v_{3}=2 \lambda_{4}+\frac{1}{2}\left(\lambda_{2}-\lambda_{1}\right)^{2} /\left(\lambda_{1}+\lambda_{2}-\right.$ $\left.2 \lambda_{4}\right)$. This yields for the velocity of the harmonic edge

$$
v_{h}=u_{L}+2 c_{L}-\frac{c_{R}^{2}}{2 c_{L}-c_{R}}
$$

In the reference frame $\mathcal{R}^{\prime}$ in which the solitonic edge is at 

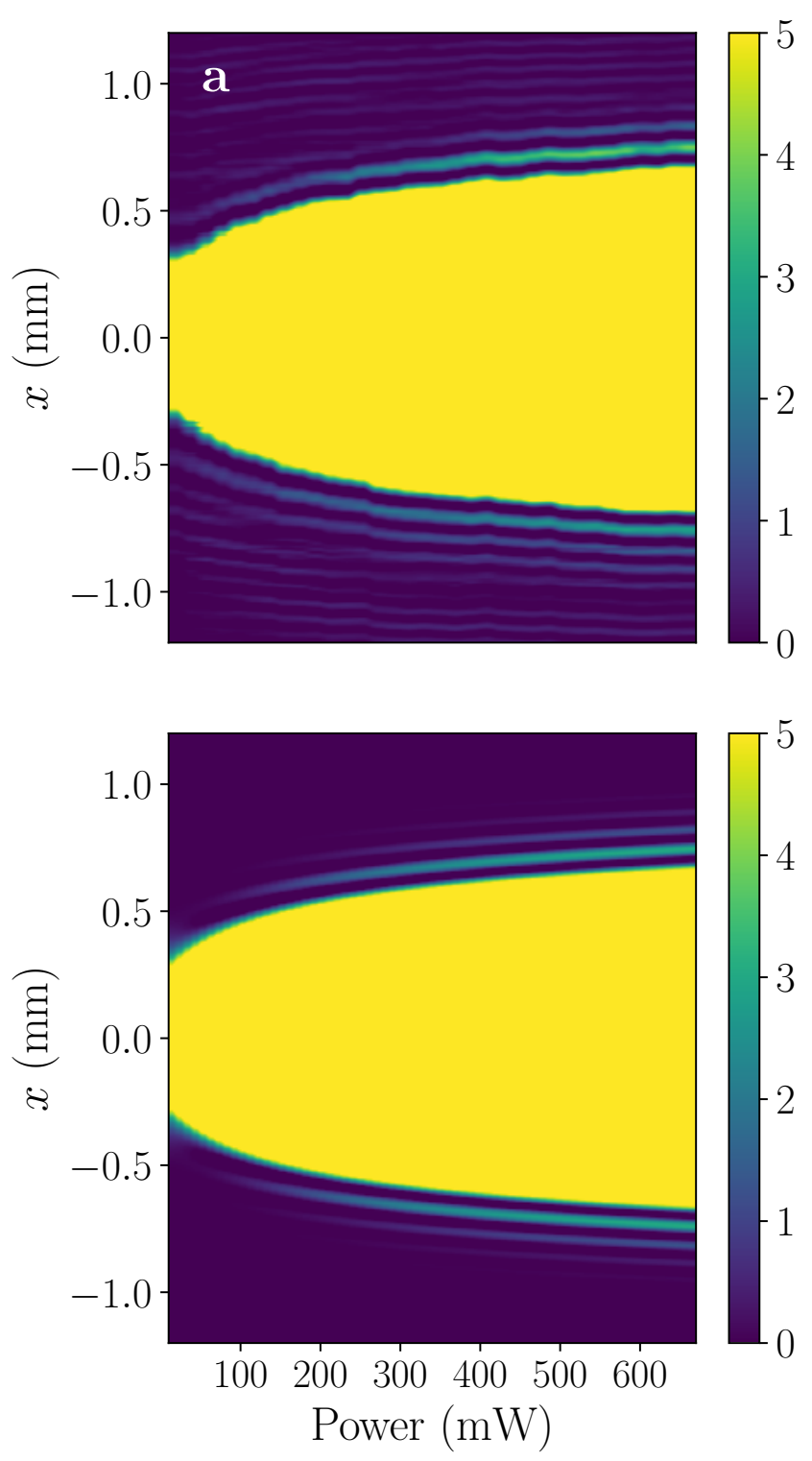

FIG. S2. Normalized intensity $\widetilde{I}_{\text {out }}(x, y=0)$ as a function of $P_{\text {tot }}$. (a) Experimental data. (b) Two-dimensional numerical simulations of Eq. (S2).

rest, the fluid of light at the right boundary $(x \rightarrow+\infty)$ propagates at velocity $u_{R}^{\prime}=u_{R}-v_{s}=-c_{L}$ : the flow is directed to the left and is supersonic (clearly $c_{L}>c_{R}$ since $I_{L}>I_{R}$ ). On the other hand, still in $\mathcal{R}^{\prime}$, the fluid at the left boundary has a velocity $u_{L}^{\prime}=u_{L}-v_{s}=c_{L}-2 c_{R}$. One can easily verify that this quantity is lower in norm than the left speed of sound $c_{L}$. If $I_{L} \geq 4 I_{R}$, the DSW admits a vacuum point [1] and the velocity $u_{L}^{\prime}$ is positive. This velocity is directed to the left only if $I_{L}<4 I_{R}$, i.e., if the mismatch in asymptotic intensities is not too large. In this case the flow in $\mathcal{R}^{\prime}$ is always directed to the left, and is upstream supersonic and downstream subsonic. This is an analogue white hole, with the important pro-
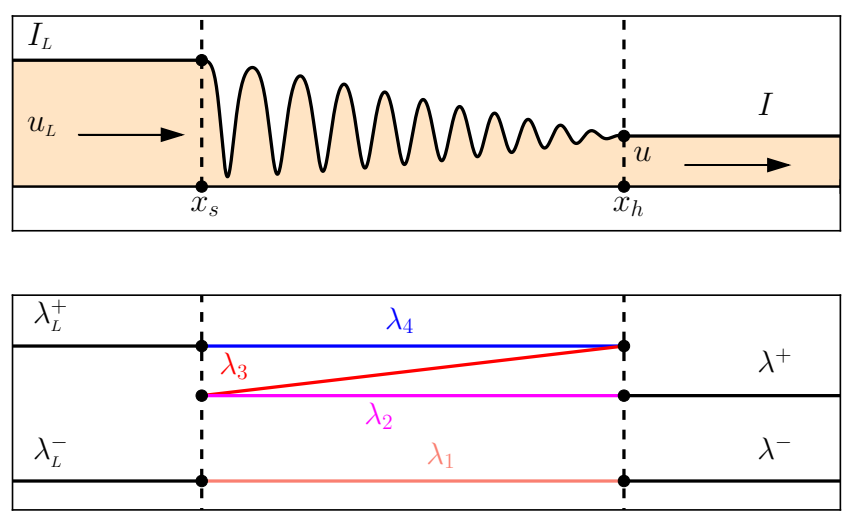

$x$

FIG. S3. Upper plot: schematic representation of the intensity pattern $I(x, t)$ in the presence of a Gurevitch-Pitaevskii DSW. Lower plot: corresponding arrangement of the Riemann invariants.

viso that the structure is not stationary: $I$ and $u$ are time-dependent in any reference frame. However, it has been established in the case of Korteweg-de Vries equation [3, 4] and also for the nonlinear Schrödinger equation $[5,6]$ that if one takes dissipation into account, after a (typically long) transient, the structure becomes stationary and the analogy with a white hole is then more straightforward.

Note however, that even in a stationary case, as for any dispersive analog, the horizon is "fuzzy", i.e., cannot be determined by the condition that the flow velocity is equal to the local sound velocity, because within the DSW the density varies over too short a distance to enable a proper definition of a local sound velocity. Actually, in the domain of analogue gravity, it is more important to correctly describe the scattering of small amplitude linear waves than to try to precisely locate the horizon. For instance the correct Hawking temperature of a dispersive analogue is determined by the low energy behavior of the scattering coefficients, and not by the characteristics of the flow at an elusive horizon. Pursuing this line of reasoning to its ultimate consequence leads to consider analogue gravity as a sub-domain of wave-mean flow interaction. In this line, it seems legitimate to follow the approach of Ref. [7] which studies the scattering of linear waves by replacing the DSW by an average mean flow. The good results obtained thanks to this method suggest a possible solution of the above discussed difficulty for locating the horizon of a DSW: a proper definition could be reached by studying the corresponding average mean flow. 
[1] G. El, V. Geogjaev, A. Gurevich, and A. Krylov, Decay of an initial discontinuity in the defocusing NLS hydrodynamics, Physica D 87, 186 (1995).

[2] A. M. Kamchatnov, Nonlinear Periodic Waves and Their Modulations-An Introductory Course (World Scientific, Singapore, 2000).

[3] A. V. Gurevich and L. P. Pitaevskii, Averaged description of waves in the Korteweg-de Vries-Burgers equation, Sov. Phys. JETP 66, 409 (1987).
[4] V. V. Avilov, I. M. Krichever, and S. P. Novikov, Evolution of Whitham zone in the theory of Korteweg-de Vries, Sov. Phys. Dokl. 32, 564 (1987).

[5] A. Kamchatnov, On Whitham theory for perturbed integrable equations, Physica D 188, 247 (2004).

[6] P.-E. Larré, N. Pavloff, and A. M. Kamchatnov, Wave pattern induced by a localized obstacle in the flow of a one-dimensional polariton condensate, Phys. Rev. B 86, 165304 (2012).

[7] T. Congy, G. A. El, and M. A. Hoefer, Interaction of linear modulated waves and unsteady dispersive hydrodynamic states with application to shallow water waves, J. Fluid Mech. 875, 1145 (2019). 